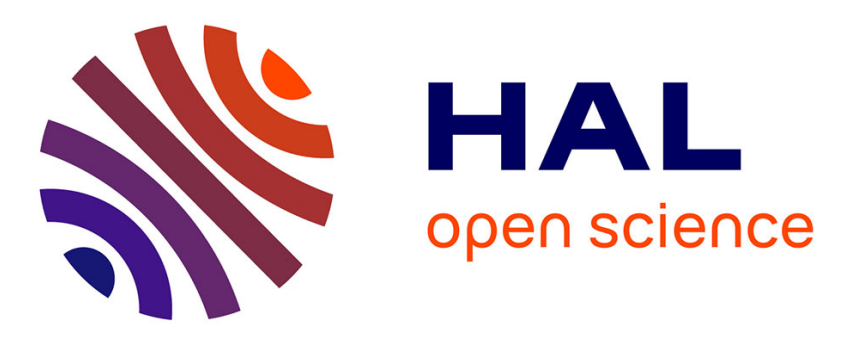

\title{
A model-based eco-routing strategy for electric vehicles in large urban networks
}

\author{
Giovanni de Nunzio, Laurent Thibault, Antonio Sciarretta
}

\section{To cite this version:}

Giovanni de Nunzio, Laurent Thibault, Antonio Sciarretta. A model-based eco-routing strategy for electric vehicles in large urban networks. 2016 IEEE 19th International Conference on Intelligent Transportation Systems (ITSC), Nov 2016, Rio de Janeiro, Brazil. pp.2301 - 2306, 10.1109/ITSC.2016.7795927 . hal-01515215

\section{HAL Id: hal-01515215 https://hal-ifp.archives-ouvertes.fr/hal-01515215}

Submitted on 27 Apr 2017

HAL is a multi-disciplinary open access archive for the deposit and dissemination of scientific research documents, whether they are published or not. The documents may come from teaching and research institutions in France or abroad, or from public or private research centers.
L'archive ouverte pluridisciplinaire HAL, est destinée au dépôt et à la diffusion de documents scientifiques de niveau recherche, publiés ou non, émanant des établissements d'enseignement et de recherche français ou étrangers, des laboratoires publics ou privés. 


\title{
A Model-Based Eco-Routing Strategy for Electric Vehicles in Large Urban Networks
}

\author{
Giovanni De Nunzio, Laurent Thibault, Antonio Sciarretta
}

\begin{abstract}
A novel eco-routing navigation strategy and energy consumption modeling approach for electric vehicles are presented in this work. Speed fluctuations and road network infrastructure have a large impact on vehicular energy consumption. Neglecting these effects may lead to large errors in eco-routing navigation, which could trivially select the route with the lowest average speed. We propose an energy consumption model that considers both accelerations and impact of the road infrastructure, also separating the costs of all the possible turning movements in the transportation network by means of the adjoint graph. The approach makes use of only aggregated data available from existing map services. We demonstrate that the proposed strategy is more effective and reliable than the existing approaches in predicting vehicle energy consumption and in suggesting an energy-efficient route.
\end{abstract}

Index Terms - Eco-routing, energy consumption estimation, electric vehicles, adjoint graph.

\section{INTRODUCTION}

While research on energy-efficient driving trajectories (i.e. eco-driving) has already witnessed numerous efforts [1], the impact analysis of route choice on energy consumption and the development of energy-efficient navigations systems (i.e. eco-routing) are relatively new subjects. Current available navigation systems suggest either the fastest or the shortest route. However, these route choices are not always the most energy-efficient ones [2], [3].

The main challenge of an eco-routing navigation system lies in the accurate estimation of the vehicle energy consumption, as demonstrated in [4], [5]. Such energy consumption should be estimated on each of the different road segments composing the routing network of the area under analysis. Models for estimating vehicle energy consumption can be broadly divided into macroscopic and microscopic models, depending on how vehicular activities are aggregated over time and space. In a comprehensive study in [5], a comparison of several existing energy consumption and emission models for combustion-engine vehicles was conducted. In general, microscopic models are considered more precise because they take as input instantaneous velocities and accelerations, which are difficult to obtain without equipped probe vehicles recording GPS or CAN-bus data. Eco-routing navigation systems based on this type of models need to acquire a large amount of driving data in order to determine a statistical cost on each road segment of the routing model.

G. De Nunzio, Department of Control, Signal and System at IFPen, RueilMalmaison, France, giovanni.de-nunzio@ifpen.fr

L. Thibault, Department of Control, Signal and System at IFPen, RueilMalmaison, France, laurent.thibault@ifpen.fr

A. Sciarretta, Department of Control, Signal and System at IFPen, RueilMalmaison, France, antonio.sciarretta@ifpen.fr
For instance, in [6] the authors propose a strategy to reconstruct synthetic speed profiles from historical speed data to calculate an instantaneous power demand and an energy cost for the road segments. In [7], analogously, the authors propose to compute eco-weights based on GPS data, and to periodically maintain these weights as new information is collected. Intuitively, this approach requires a long time to have a reliable and dense mapping of the energy costs of all the road segments in the routing network.

On the other hand, macroscopic models take as input mean velocities, mean travel time and road grade, which are typically easier to obtain through free or commercial historical databases. Such aggregated models can be further classified into regression-based models and load-based models. The regression-based models are generally difficult to calibrate in order for all the parameters of the model to be significant, and they may lead to random errors, which are not easy to explain due to the little physical foundation of these models [8], [9], [10]. The load-based models rely upon the longitudinal dynamics of the vehicle and are easier to calibrate using the vehicle construction parameters [11], [12].

All these models make strong assumptions for the energy consumption estimation, by neglecting accelerations, or road grade, or the impact of the road network topology and signalization. Only few recent works address the importance of considering accelerations even when using macroscopic models, as well as considering the elements of the road infrastructure that may cause vehicular speed disruptions. In [13], the authors propose a method to consider interlink accelerations induced by the different adjacent average speeds. The costs for all turning movements available at a certain intersection are separated. However, the scalability issue of the routing problem is not addressed.

In addition, since electric vehicles and energy recovery phenomena are considered, the eco-routing strategy has to deal with the challenge of negative energy weights. Optimal path-searching algorithms on graphs with negative weights (e.g. Bellman-Ford algorithm [14]) are often discarded in literature because of their computational cost. Alternative approaches have been proposed to tackle this issue, mostly based on heuristics for graph weights shifting [12] or nonoptimal path searching [15].

In this work, we propose a novel macroscopic energy consumption model and a novel eco-routing strategy. The novelty of the proposed approach is summarized as follows. First, our macroscopic load-based energy consumption model considers accelerations to move from one road segment to the adjacent ones. Also, additional accelerations induced by 
the road topology and infrastructure (e.g. traffic lights, stop signs, turns, etc.) are considered in the energy consumption model. Then, since considering accelerations introduces a correlation between the energy cost of adjacent links, and the cost may not be unique due to the presence of multiple turning movements, the routing strategy is deployed on the weighted adjoint graph. This solves the size issues mentioned in [13], because all the turning movements are decoupled avoiding the exponential growth of the routing graph. Finally, an optimal path-searching algorithm for graphs with negative weights is used, the computational time is showed to remain acceptable, and a strategy for a real-time use of the ecorouting navigation system is suggested.

The problem is presented and formulated in Section II. The simulation and experimental results are illustrated in Section III. Concluding remarks are given in Section IV.

\section{PROBLEM FORMULATION}

Eco-routing navigation aims to find the most energyefficient route in a vehicular road network to travel from an origin to a destination. It may be seen as an optimization problem where the function to be minimized is the energy consumption of the vehicle.

In the following we will introduce and detail the different elements of the proposed eco-routing strategy.

\section{A. Vehicle Energy Consumption Model}

Our analysis focuses mainly on the eco-routing for electric vehicles, therefore the energy consumption model is required to capture regenerative braking, as well as electric drive efficiency.

The vehicle longitudinal dynamical model may be generally written as [16]:

$$
m \frac{d v(t)}{d t}=F_{w}-F_{\text {aero }}-F_{\text {friction }}-F_{\text {slope }}
$$

where $m$ is the vehicle mass, $v(t)$ the vehicle speed, $F_{w}$ the force at the wheels, $F_{\text {aero }}$ the aerodynamic force, $F_{\text {friction }}$ the rolling resistance force, $F_{\text {slope }}$ the gravity force.

Therefore, the vehicle model shall be written as:

$$
\left\{\begin{array}{l}
\dot{x}(t)=v(t) \\
m \dot{v}(t)=F_{w}-\frac{1}{2} \rho_{a} A_{f} c_{d} v(t)^{2}-m g c_{r}-m g \sin (\alpha(x))
\end{array}\right.
$$

where $\rho_{a}$ is the external air density, $A_{f}$ is the vehicle frontal area, $c_{d}$ is the aerodynamic drag coefficient, $c_{r}$ is the rolling resistance coefficient, $\alpha(x)$ is the road slope as a function of the position, and $g$ is the gravity.

The sum of the aerodynamic and rolling frictions, namely the road load force, is often approximated as a second order polynomial in the speed $v$ :

$$
F_{\text {res }}=F_{\text {aero }}+F_{\text {friction }}=a_{2} v(t)^{2}+a_{1} v(t)+a_{0}
$$

where $a_{0}, a_{1}$ and $a_{2}$ are parameters identified for a considered vehicle.

Therefore, the force at the wheels can be expressed as:

$$
F_{w}=m \dot{v}(t)+a_{2} v(t)^{2}+a_{1} v(t)+a_{0}+m g \sin (\alpha(x))
$$

The torque requested from the electric motor to meet the force demand at the wheels is defined as:

$$
T_{m}= \begin{cases}\frac{F_{w} r}{\rho_{t} \eta_{t}}, & \text { if } F_{w} \geq 0 \\ \frac{F_{w} r \eta_{t}}{\rho_{t}}, & \text { if } F_{w}<0\end{cases}
$$

where $r$ is the wheel radius, $\rho_{t}$ and $\eta_{t}$ are the transmission ratio and efficiency, respectively.

The torque generated by the electric motor is saturated by a maximum torque $T_{m, \max }$ and a minimum torque $T_{m, \min }$. In particular, during braking phases, if the motor torque is less negative than the negative saturation $T_{m \text {,min }}$, then the vehicle is slowed down only by the regenerative brake. Otherwise, the mechanical brake works together with the regenerative brake.

The power available at the electric motor shaft, in the presence of regenerative braking mechanism, shall be written as follows:

$$
P_{m}=\left\{\begin{array}{l}
T_{m, \text { max }} \cdot \omega(t), \quad \text { if } T_{m} \geq T_{m, \text { max }} \\
T_{m} \cdot \omega(t), \quad \text { if } T_{m, \text { min }}<T_{m}<T_{m, \text { max }} \\
T_{m, \text { min }} \cdot \omega(t), \quad \text { if } T_{m} \leq T_{m, \text { min }}
\end{array}\right.
$$

where $\omega(t)$ is the motor rotational regime and is defined as:

$$
\omega(t)=\frac{v(t) \rho_{t}}{r}
$$

Finally, the power demand at the battery of the electric vehicle, can be expressed as:

$$
P_{b}= \begin{cases}\frac{P_{m}}{\eta_{b}}, & \text { if } P_{m} \geq 0 \\ P_{m} \eta_{b}, & \text { if } P_{m}<0\end{cases}
$$

where $\eta_{b}$ is the electric drive overall efficiency, here assumed to be the same both in motor and generator mode.

Therefore, the battery energy consumption over the generic travel time $T$ is obtained as:

$$
E_{b}=\int_{0}^{T} P_{b} \mathrm{~d} t
$$

\section{B. Eco-Routing Model}

The road transportation network can be modeled as a graph. Let $\mathcal{G}=(V, A)$ be such a graph, where $V$ is the set of vertices (or nodes) of cardinality $n$, and $A$ is the set of feasible arcs (or links) of cardinality $m$ connecting the nodes of the graph. Let us define a weighting function $w: A \rightarrow W$, which associates each link of the graph with a weight.

In conventional routing graphs, the weight associated with each arc is either the length of the arc or its travel time. In the eco-routing framework, each link of the graph is assigned a weight that represents the travel energy expenditure.

The objective of this work is to design a reliable ecorouting strategy solely based on statistical and topological information about the road transportation network, without any use of real driving data. This kind of information, although often incomplete and/or imprecise, is generally 
available through commercial maps web-services. For each link $i \in A$ of the graph, it is possible to know the link length $l_{i}$, a prevailing average traffic speed $\bar{v}_{i}$ which depends on the time of the day, and the road grade $\alpha_{i}(x)$ which varies within the considered link depending on the position.

Note that a time-variant speed or acceleration profile is not available through the maps web-services, and the energy consumption model described in Section II-A cannot be directly used to assign the weights to each link of the graph.

1) Modified Energy Consumption Model: The model equations will be rewritten by replacing the time-variant speed $v(t)$ with the average traffic speed $\bar{v}$. All the vehicles on link $i$ are supposed to travel at speed $\bar{v}_{i}$. Therefore, the force expression in (4) is modified for each link $i$ as follows:

$$
\bar{F}_{w, i}=a_{2} \bar{v}_{i}^{2}+a_{1} \bar{v}_{i}+a_{0}+m g \sin \left(\alpha_{i}(x)\right)
$$

with no acceleration term. The motor torque becomes:

$$
\bar{T}_{m, i}= \begin{cases}\frac{\bar{F}_{m, i} r}{\rho_{t} \eta_{t}}, & \text { if } \bar{F}_{w, i} \geq 0 \\ \frac{\bar{F}_{m, i} r \eta_{t}}{\rho_{t}}, & \text { if } \bar{F}_{w, i}<0\end{cases}
$$

The electric motor rotational regime is also constant over time if constant speed is assumed:

$$
\bar{\omega}_{i}=\frac{\bar{v}_{i} \rho_{t}}{r}
$$

The mechanical power available at the electric motor is rewritten as:

$$
\bar{P}_{m, i}=\left\{\begin{array}{l}
T_{m, \max } \cdot \bar{\omega}_{i}, \quad \text { if } \bar{T}_{m, i} \geq T_{m, \max } \\
\bar{T}_{m, i} \cdot \bar{\omega}_{i}, \quad \text { if } T_{m, \min }<\bar{T}_{m, i}<T_{m, \max } \\
T_{m, \min } \cdot \bar{\omega}_{i}, \quad \text { if } \bar{T}_{m, i} \leq T_{m, \min }
\end{array}\right.
$$

In the following, it is assumed that the saturation torque is independent from the motor regime. Finally, the power demand at the battery of the electric vehicle, considering the electric drive efficiency $\eta_{b}$ constant, can be rewritten as:

$$
\bar{P}_{b, i}= \begin{cases}\frac{\bar{P}_{m, i}}{\eta_{b}}, & \text { if } \bar{P}_{m, i} \geq 0 \\ \bar{P}_{m, i} \eta_{b}, & \text { if } \bar{P}_{m, i}<0\end{cases}
$$

and ultimately, the battery energy consumption is:

$$
\bar{E}_{b, i}=\bar{P}_{b, i} T_{i}
$$

where $T_{i}=l_{i} / \bar{v}_{i}$ is the travel time on link $i$ when traveling at the average traffic speed $\bar{v}_{i}$.

2) Interface Accelerations: In order to take into account the acceleration effects and improve the model and the energy consumption estimation, the trip on each link is supposed to be composed of two phases: a cruising phase at the constant speed $\bar{v}_{i}$ and an acceleration phase to go from $\bar{v}_{i-1}$ to $\bar{v}_{i}$.

The energy consumption associated with the change of velocity at a node between two links is defined as:

$$
E_{\text {jump }, i}=\int_{0}^{t_{\text {jump }, i}} \tilde{P}_{b, i} \mathrm{~d} t
$$

where the battery power demand $\tilde{P}_{b, i}$ is derived analogously from an interface force at the wheels defined as:

$$
\tilde{F}_{w}=m \cdot a+a_{2} v(t)^{2}+a_{1} v(t)+a_{0}
$$

The time-varying velocity $v(t)$ in every transient is linearly modeled as $v(t)=\bar{v}_{i-1}+\operatorname{sign}\left(\bar{v}_{i}-\bar{v}_{i-1}\right) \cdot a \cdot t$, where $\bar{v}_{i-1}$ is the constant velocity on the incoming link, $\bar{v}_{i}$ the constant velocity on the outgoing link, and $a$ the constant acceleration to perform the jump. The speed change is performed in:

$$
t_{\text {jump }, i}=\frac{\bar{v}_{i}-\bar{v}_{i-1}}{\operatorname{sign}\left(\bar{v}_{i}-\bar{v}_{i-1}\right) \cdot a}
$$

Therefore, the total energy consumption on link $i$ can be rewritten as:

$$
E_{b, i}=\bar{E}_{b, i}+E_{\text {jump }, i}
$$

As mentioned before, a limitation of this modeling solution is that speed fluctuations within a link are neglected, which could be an issue in traffic. However, modeling the acceleration within a link seems unrealistic at a route planning level, because it highly depends on local traffic conditions, and as such is often spontaneous and unpredictable [13].

3) Impact of the Infrastructure: The consideration of interface accelerations allows the energy consumption model to be more precise and realistic. However, as mentioned before, the available a-priori information about the average traffic speed is not always complete nor updated. In particular, it is very difficult and unlikely to have accurate speed information on secondary streets. This may lead to underestimations of the energy consumption and, therefore, lead the eco-routing algorithm to blindly favor this type of streets.

Disruptions in the speed profiles and accelerations are caused not only by traffic, but also by the infrastructure. In particular, critical elements of the road infrastructure, such as traffic lights, intersections, and turning movements are very likely to induce stops or significant decelerations.

Therefore, when the information about the position of these critical elements is available, the energy consumption term associated with acceleration in (16) should be modified.

For instance, the speed change between two links connected by a stop sign will be modeled as two distinct transients: the first transient from $\bar{v}_{i-1}$ to 0 , the second transient from 0 to $\bar{v}_{i}$. Therefore, the energy consumption associated with the speed change at the node can be split in:

$$
E_{\text {jump }, i}=\int_{0}^{t_{\text {jump } 1, i}} \tilde{P}_{b 1, i} \mathrm{~d} t+\int_{0}^{t_{\text {jump } 2, i}} \tilde{P}_{b 2, i} \mathrm{~d} t
$$

where the speed variation in the first term is modeled as $v_{1}(t)=\bar{v}_{i-1}-a \cdot t$, the time to perform the first transient is $t_{\text {jump } 1, i}=\bar{v}_{i-1} / a$, the speed variation in the second term is modeled as $v_{2}(t)=a \cdot t$, and the time to perform the second transient is $t_{\text {jump } 2, i}=\bar{v}_{i} / a$.

4) Adjoint Graph: The consideration of the interface accelerations between adjacent links arises a major issue in the graph modeling of the road network. In particular, every node of the graph with two or more incoming links is critical 
because $\bar{v}_{i-1}$ and, consequently, $E_{\text {jump }, i}$ are not unique. Evidently, this prevents from assigning unique energy costs to the links of the graph. Hence, the graph $\mathcal{G}$ is not adequate for the proposed energy modeling approach. This difficulty is resolved by introducing the adjoint graph and by solving the routing problem on the adjoint graph instead of graph $\mathcal{G}$.

The adjoint graph $\mathcal{L}(\mathcal{G})$ of a graph $\mathcal{G}$ has as nodes the links of $\mathcal{G}$, and two nodes of $\mathcal{L}(\mathcal{G})$ are adjacent whenever the corresponding links of $\mathcal{G}$ are adjacent. The in-degree $\operatorname{id}\left(n_{j}\right)$ of a node $n_{j} \in V$ is the number of links entering $n_{j}$, while its out-degree od $\left(n_{j}\right)$ is the number of links leaving $n_{j}$.

Let $\mathcal{G}=(V, A)$ be the graph with $n$ nodes and $m$ arcs as before. Then $\mathcal{L}(\mathcal{G})=\left(V^{*}, A^{*}\right)$ is the adjoint graph with $n^{*}=m$ nodes, and $m^{*}=\sum_{j=1}^{n}\left[\operatorname{id}\left(n_{j}\right) \cdot \operatorname{od}\left(n_{j}\right)\right]$ links.

Each link $i \in A$ of the original graph $\mathcal{G}$ becomes a node $i \in V^{*}$ of the adjoint graph $\mathcal{L}(\mathcal{G})$. We can define as before an in-degree $\operatorname{id}(i)$ and an out-degree $\operatorname{od}(i)$ for node $i \in V^{*}$.

Let us then define a weighting function for the links of the adjoint graph $w^{*}: A^{*} \rightarrow W^{*}$, where the weight for each link $k \in A^{*}$ is:

$$
W_{k}^{*}=\left\{\begin{array}{l}
\bar{E}_{b, i}+E_{\text {jump }, i}, \text { if } \operatorname{id}(i-1) \neq 0 \\
\bar{E}_{b, i}+E_{\text {jump }, i}+\bar{E}_{b, i-1}, \text { if } \operatorname{id}(i-1)=0
\end{array}\right.
$$

By using the adjoint graph $\mathcal{L}(\mathcal{G})$ as routing graph, the energy weights are uniquely assigned to each link of the graph by decoupling all the possible turning movements modeled in the original graph $\mathcal{G}$.

Note that our objective in this framework is to address solely energy aspects, and therefore the weight assigned to each link of the adjoint graph represents only the associated energy consumption. The framework can be easily extended to consider also travel time, and the optimization would search then for a trade-off solution between energy consumption and travel time minimization.

\section{Shortest Path Algorithm}

Finally, the last stage of the problem formulation consists in the actual minimization of the energy consumption to travel from a selected origin to a destination in the road network. Minimization of a cost in a graph can be solved by means of a standard shortest path algorithm.

Such algorithm will be run on the adjoint graph. Note that the weights on the arcs can be negative, since regenerative braking is considered. Therefore, the Bellman-Ford algorithm is a necessary choice to find the global optimum [14]. The design and functioning scheme of the proposed eco-routing strategy is summarized in Fig. 1.

\section{EXPERIMENTS}

The simulation study and the experimental campaign were conducted on the road network of the city center of Lyon (i.e. about 5,400 nodes and 9,500 links), in France. The webservice HERE Maps [17] was used in the experiments for obtaining information about network topology, road grade, current traffic conditions, and critical elements of the road infrastructure as previously discussed.

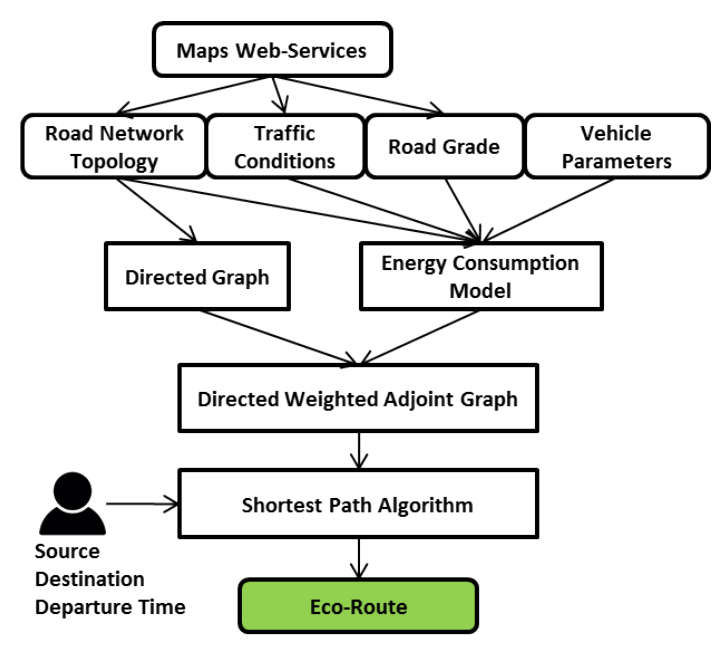

Fig. 1: Overview of the proposed eco-routing strategy.

The road grade and the road network topology, along with traffic lights position, are supposed not to vary with time. The traffic conditions, on the contrary, are extremely timedependent. An ideal real-time query on the web-service to retrieve the traffic conditions of the entire road network is not suitable for online use of the eco-routing navigation system.

A solution, more suitable for online purposes, may be to store offline the global information about historical traffic conditions for different days of the week and for different times of the day. The real-time adaptation is carried out only after the driver selects the desired origin, destination and departure time. The total cost of only the historical $K$ best eco-routes is updated according to the current traffic conditions, and compared in order to determine the current best itinerary in terms of energy consumption.

The vehicle parameters are reported in Table I.

TABLE I: Vehicle parameters

\begin{tabular}{l|c|l}
\hline Description & Parameter & \multicolumn{1}{|c}{ Value } \\
\hline Vehicle mass & $m$ & $1190 \mathrm{~kg}$ \\
Tire radius & $r$ & $0.2848 \mathrm{~m}$ \\
Transmission ratio & $\rho_{t}$ & 5.763 \\
Transmission efficiency & $\eta_{t}$ & 0.95 \\
Acceleration & $a$ & $1.5 \mathrm{~m} / \mathrm{s}^{2}$ \\
Coefficient & $a_{0}$ & $125.73 \mathrm{~N}$ \\
Coefficient & $a_{1}$ & $1.72 \mathrm{~N} /(\mathrm{m} / \mathrm{s})$ \\
Coefficient & $a_{2}$ & $0.58 \mathrm{~N} /(\mathrm{m} / \mathrm{s})^{2}$ \\
Motor minimum torque & $T_{m, \min }$ & $-50 \mathrm{Nm}$ \\
Motor maximum torque & $T_{m, \max }$ & $200 \mathrm{Nm}$ \\
Electric drive efficiency & $\eta_{b}$ & 0.85 \\
\hline
\end{tabular}

\section{A. Simulation Study: Eco-Route Performance}

The first set of simulation experiments aims to compare the eco-route suggested by our navigation system with the shortest and the fastest routes. In particular, the performance analysis has been conducted in terms of energy consumption computed by the proposed model and travel time. An energy consumption ratio (i.e. ratio between the energy consumption of the eco-route and the shortest or fastest route) and a travel 

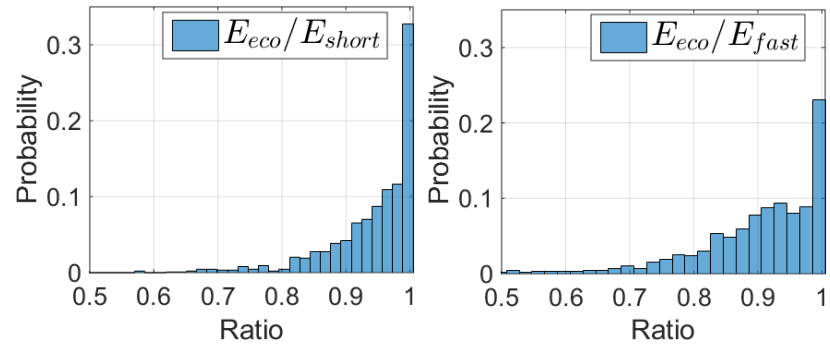

(a) Histogram Eco vs. Shortest

(b) Histogram Eco vs. Fastest

Fig. 2: Energy consumption ratio probability distribution.

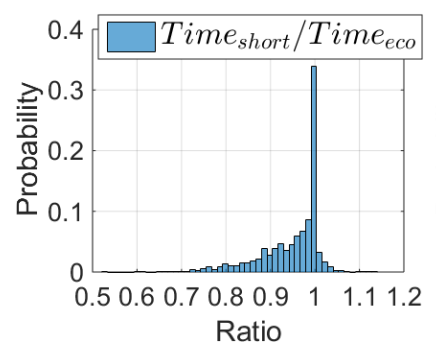

(a) Histogram Shortest vs. Eco

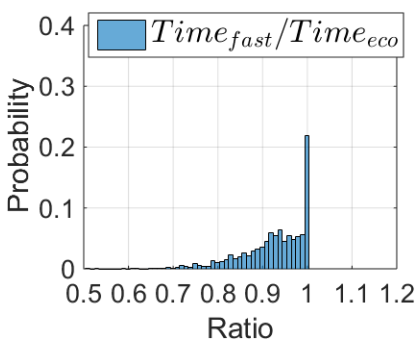

(b) Histogram Fastest vs. Eco
Fig. 3: Travel time ratio probability distribution.

time ratio (i.e. ratio between the travel time of the fastest route and the eco or shortest route) are evaluated.

For a random selection of 1,000 different origindestination (O/D) pairs in the considered road network, the shortest-path algorithm has been run on the adjoint graph weighted with the energy consumption to compute the ecoroute, and on the original graph weighted with link length and travel time to compute the shortest-route and fastestroute. The Bellman-Ford algorithm convergence time was 0.02 seconds in average on a laptop computer equipped with a CPU at $2.8 \mathrm{GHz}$ and $16 \mathrm{~GB}$ of RAM. For the sake of comparison, traffic conditions have been fixed at a specific date and time, namely at peak hour of a working day.

In Fig. 2 the probability distribution of the energy consumption ratio is reported. The energy consumption predicted by the model for the eco-route has been compared to the energy consumption of the shortest and fastest itinerary, respectively. The probability distribution of the energy gain provided by the eco-route with respect to the shortest route is represented in Fig. 2a. The mean value of the distribution is $\mu=0.94$, and the standard deviation is $\sigma=0.07$. Therefore, the eco-route shows an average energy saving of about $6 \%$ with respect to the shortest route. The probability distribution of the energy gain with respect to the fastestroute is represented in the histogram in Fig. 2b. The mean value of the distribution is $\mu=0.9$, and the standard deviation is $\sigma=0.1$. So, the eco-route shows an average energy saving of about $10 \%$ with respect to the fastest route.

An analogous analysis has been conducted on the travel time performance of the three itineraries calculated for each O/D pair, as reported in Fig. 3. The probability distribution of the travel time performance provided by the shortest-route with respect to the eco-route is represented in Fig.3a. The mean value of the distribution is $\mu=0.95$, and the standard deviation is $\sigma=0.07$. Therefore, the shortest-route shows an average travel time improvement of about $5 \%$ with respect to the eco-route. The probability distribution of the travel time performance provided by the fastest-route with respect to the eco-route is represented in Fig. 3b. The mean value of the distribution is $\mu=0.93$, and the standard deviation is $\sigma=0.07$. Therefore, the fastest-route shows an average travel time improvement of about $7 \%$ with respect to the ecoroute. Note that the shortest-route can also present a worse performance than the eco-route in terms of travel time.

\section{B. Experimental Campaign: Validation Results}

A validation analysis was carried out in order to verify the accuracy of the proposed energy consumption model and, therefore, the reliability of the eco-routing navigation results.

In order to perform the validation analysis, several real driving profiles, consisting of GPS coordinates and driving speed, were recorded during an experimental campaign in the city center of Lyon. The driving profiles used for validation were all recorded at the same time of the day on different dates. The energy consumption associated with the real driving profiles was calculated using the instantaneous model described in Section II-A. The eco-routing model was constructed using the average traffic speed of a sample working day at the same time of the tests.

In the following, the validation results of two driving tests are reported. The first driving test was conducted on a combination of urban roads and highway. The second driving test was conducted on a secondary urban road. In the first test, as shown in Fig. 4, the real driving speed profiles in the dotted blue lines show good repeatability characteristics, though recorded on different days. This translates into a similar trip energy consumption, as shown in Fig. 5, where the cumulative energy expenditure is represented as a function of the traveled distance. The average value of the real energy consumption (i.e. dotted blue lines) at the end of the trip is $296.4 \mathrm{Wh}$, and the standard deviation is $12.5 \mathrm{Wh}$. The proposed energy consumption model, in green in Fig. 5, overestimates the total energy consumption by $7 \%$ with respect to the average of the real consumption. In order to further assess the performance and the advantages of the proposed strategy, the current state-of-the-art eco-routing approach based only on the average traffic speed was also tested on the validation test. As shown in magenta in Fig. 5, the energy consumption model based solely on the average traffic speed makes an estimation error of $32 \%$.

Similar results can be observed on the second validation test, as shown in Fig. 6 and 7. The average value of the real energy consumption is $246.3 \mathrm{Wh}$, and the standard deviation is $7.8 \mathrm{Wh}$. The proposed energy consumption model, in green in Fig. 7, underestimates the total energy consumption by 9\%. On the contrary, the energy consumption model based solely on the average traffic speed makes a significantly larger error of $38 \%$. Such model is not able to properly capture all the stops and accelerations of the real driving profiles in Fig. 6. The proposed model is more robust to imperfect information, thanks to the consideration of 


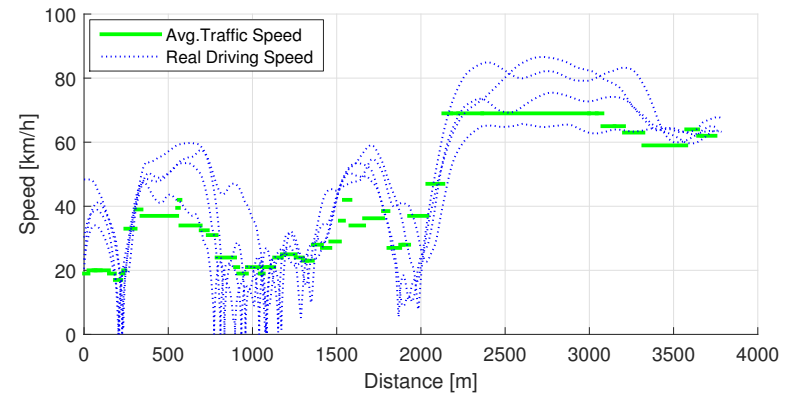

Fig. 4: Test 1: driving speed profiles and mean traffic speed.

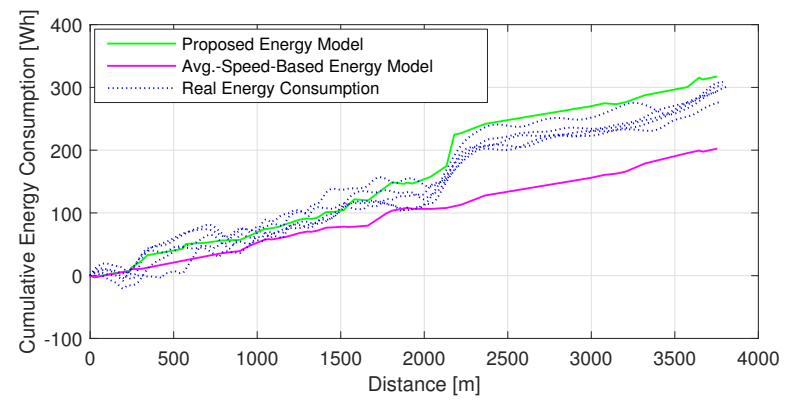

Fig. 5: Test 1: comparison between the real cumulative energy consumption and the energy consumption estimation.

interface accelerations and infrastructure critical points that induce stops and additional accelerations.

\section{CONCLUSIONS}

A novel model-based eco-routing strategy for electric vehicles in large urban networks was proposed. The energy consumption model takes into account accelerations at the interface between adjacent road segments, as well as additional acceleration terms induced by critical road infrastructure elements. The introduction of the adjoint graph allowed for a correct and accurate energy modeling of the routing network. The proposed strategy exploits only information available online, without any model refinement by means of userrecorded experimental data. Future integration of travel time terms in the optimization framework, in order to discard impractical and unappealing eco-routes, is under study.

\section{ACKNOWLEDGMENTS}

This project has received funding from the European Union's Horizon 2020 research and innovation program under grant agreement No. 653288 - OPTEMUS.

\section{REFERENCES}

[1] A. Sciarretta, G. De Nunzio, and L. L. Ojeda, "Optimal Ecodriving Control: Energy-Efficient Driving of Road Vehicles as an Optimal Control Problem," IEEE Control Systems Magazine, vol. 35, no. 5, pp. 71-90, 2015.

[2] K. Ahn and H. Rakha, "The Effects of Route Choice Decisions on Vehicle Energy Consumption and Emissions," Transportation Research Part D, vol. 13, pp. 151-167, 2008.

[3] E. Ericsson, H. Larsson, and K. Brundell-Freij, "Optimizing Route Choice for Lowest Fuel Consumption Potential Effects of a New Driver Support Tool," Transportation Research Part C, vol. 14, pp. 369-383, 2006.

[4] M. Kubička, J. Klusáček, A. Sciarretta, A. Cela, H. Mounier, L. Thibault, and S.-I. Niculescu, "Performance of Current Eco-Routing Methods," in IEEE Intelligent Vehicles Symposium, 2016.

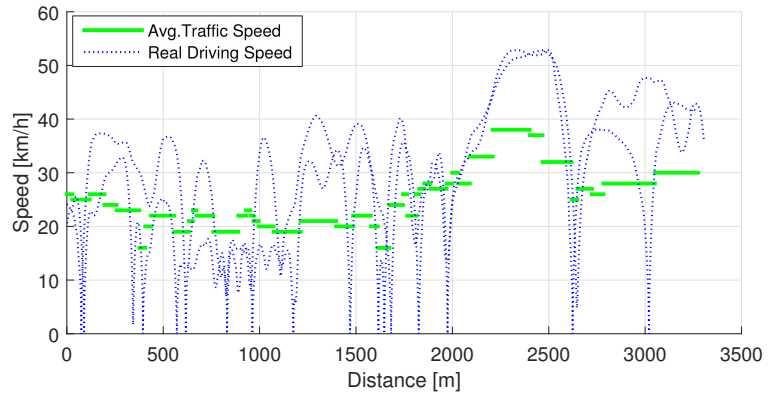

Fig. 6: Test 2: driving speed profiles and mean traffic speed.

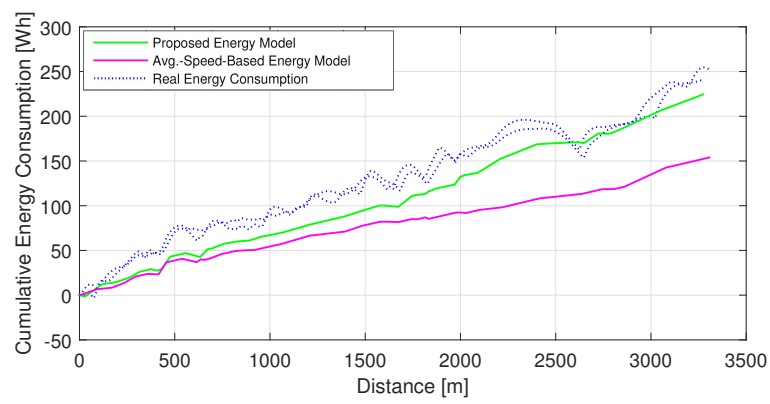

Fig. 7: Test n.2: comparison between the real cumulative energy consumption and the energy consumption estimation.

[5] C. Guo, B. Yang, O. Andersen, C. S. Jensen, and K. Torp, "EcoMark 2.0: Empowering Eco-Routing with Vehicular Environmental Models and Actual Vehicle Fuel Consumption Data," Geoinformatica, vol. 19, pp. 567-599, 2015

[6] C. F. Minett, A. Maria Salomons, W. Daamen, B. van Arem, and S. Kuijpers, "Eco-Routing: Comparing the Fuel Consumption of Different Routes between an Origin and Destination Using Field Test Speed Profiles and Synthetic Speed Profiles," in IEEE Forum on Integrated and Sustainable Transportation Systems, 2011, pp. 32-39.

[7] C. Guo, B. Yang, O. Andersen, C. S. Jensen, and K. Torp, "EcoSky : Reducing Vehicular Environmental Impact Through Eco-Routing," in IEEE 31st International Conference on Data Engineering, 2015, pp. 1412-1415.

[8] K. Ahn and H. A. Rakha, "Network-Wide Impacts of Eco-Routing Strategies : A Large-Scale Case Study," Transportation Research Part $D$, vol. 25, pp. 119-130, 2013.

[9] K. Boriboonsomsin, M. J. Barth, W. Zhu, and A. Vu, "Eco-Routing Navigation System Based on Multisource Historical and Real-Time Traffic Information," IEEE Transactions on Intelligent Transportation Systems, vol. 13, no. 4, pp. 1694-1704, 2012.

[10] E. Yao and Y. Song, "Study on Eco-Route Planning Algorithm and Environmental Impact Assessment," Journal of Intelligent Transportation Systems, vol. 17, no. 1, pp. 42-53, 2013.

[11] M. Richter, S. Zinser, and H. Kabza, "Comparison of Eco and Time Efficient Routing of ICEVs, BEVs and PHEVs in Inner City Traffic," in IEEE Vehicle Power and Propulsion Conference, 2012, pp. 11651169.

[12] T. Jurik, A. Cela, R. Hamouche, R. Natowicz, A. Reama, S. I. Niculescu, and J. Julien, "Energy Optimal Real-Time Navigation System," IEEE Intelligent Transportation Systems Magazine, vol. 6, no. 3, pp. 66-79, 2014.

[13] Y. M. Nie and Q. Li, "An Eco-Routing Model Considering Microscopic Vehicle Operating Conditions," Transportation Research Part $B$, vol. 55, pp. 154-170, 2013.

[14] R. Bellman, "On a Routing Problem," Quarterly of Applied Mathematics, vol. 16, no. 1, pp. 87-90, 1958.

[15] R. Abousleiman and O. Rawashdeh, "Energy-Efficient Routing for Electric Vehicles using Metaheuristic Optimization Frameworks," in 17th IEEE Mediterranean Electrotechnical Conference, 2014, pp. 298304.

[16] L. Guzzella and A. Sciarretta, Vehicle Propulsion Systems. SpringerVerlag Berlin Heidelberg, 2013.

[17] "HERE Maps." [Online]. Available: https://company.here.com/here/ 